\title{
Biologia de anofelinos amazônicos. IV. Observaçōes sobre a atividade de picar de Anopheles nuneztovari Gabaldon (Diptera, Culicidae) (1)
}

\author{
Wanderli Pedro Tadei $\left({ }^{2}\right)$ \\ José Marinho Correia $\left(^{(3)}\right.$
}

\begin{abstract}
Resumo
São relatadas observações sobre a atividade de picar de populações de Anopheles nuneztovari que apresentam hábito exofilico e cujas coletas foram feitas no período entre 18:00 e 22:00 horas, em d ferentes pontos da Rodovia BR-174 (Manaus/Boa Vist I). Foi verificado que ocorrem variaçōes no início e $t$ irmino da atividade de picar, contudo a mesma está c incentrada no horário entre 18:30 e 19:10 horas aproxir adamente.
\end{abstract}

Estudos relacionados com as d :erenças na capacidade vetora da malária hum na, em espécies do gênero Anopheles, têl mostrado que $\in$ sta condição decorre com fr qüência do fato de existirem espécies críptici , ao longo de sua área de distribuição geogr ica.

Anopheles nuneztovari, do sub inero Nyssorhynchus, ocorre nos países c norte da América do Sul, Bacia Amazônica rasil), Bolívia, Peru e Equador; contudo é nsiderada vetor primário da malária humaná penas no oeste da Venezuela e norte da Cc nbia (Gabaldon \& Guerrero, 1959; Gabaldon al., 1963; Elliott, 1968, 1972; Gabaldon, 1969 .

Kitzmiiler et al. (1973), estudé , a constituição cromossômica de populaçoes de Anopheles nuneztovari do Brasil, Venezuela e Colômbia, verificaram que a população Venezuelana-Colombiana, vetora da malária, pode ser diferenciada da população brasileira, não vetora, por uma inversão no estado homozigoto no cromossomo X. Esses autores assinalaram que A. nuneztovari parece exibir uma quantidade considerável de variabilidade em diferentes partes de sua área de ocorrência. Assinalaram também que um dos aspectos dessa variabilidade é o fato de, aparentemente, afetar a capacidade vetora da espécie. Steiner et al.
(1980) estudaram diferentes populações de A. nuneztovari quanto à variabilidade enzimática e verificaram níveis relativamente altos de variação genética. No entanto, pouca diferenciação foi observada entre as populações cromossomicamente distintas e sugeriram que a divergência pudie ser um evento relativamente recente no processo evolutivo de $A$. nuneztovari.

O comportamento de populações de Anopheles nuneztovari foi estudado por diferentes autores. Foram registradas variações quanto aos hábitos exofílico e endofílico, ao longo da área de ocorrência da espécie (Deane et al., 1948; Garcia-Martin, 1955; Gabaldon \& Guerrero, 1959; Gabaldon et al., 1963). Elliott (1968, 1972) estudando diferentes aspectos do contato homem-vetor, sugeriu que $A$. nuneztovari é composta de, pelo menos, duas espécies crípticas que apresentam diferentes padrões na atividade de picar: uma, procura preferencialmente animais ao por do sol, e em geral fora das casas, e a outra, pica o homem torde da noite e principalmente dentro das casas. Esta última é considerada o principal vetor da malária humana e ocorre no oeste da Venezuela e norte da Colômbia. As duas populações são supostamente simpátricas em algumas partes dessas regiōes.

Coletas realizadas nas proximidades da Rodovia BR-174 (Manaus/Boa Vista), desde o $\mathrm{Km} 137$ ao 530 , em janeiro e fevereiro de 1980 , possibilitaram registrar a ocorrência de populações de Anopheles nuneztovari que apresentam hábito exofílico e cuja atividade de picar o homem ocorre ao pôr do sol. Foram realizadas caputras extradomiciliares em 9 pontos da rodovia (figura 1) no horário das $18: 00$ às

(1) - Trabalho subvencionado pelo CNPq e pela FAPESP.

(2) - Instituto de Biociências (UNESP), São José do Rio Preto, SP.

(3) - Instituto Nacional de Pesquisas da Amazônia, Manaus. 
22:00 horas. Tentativas de capturas intradomiciliares também foram realizadas em 3 pontos ( $\mathrm{Km} 138$, Aeroporto da $\mathrm{FAB}$ e $\mathrm{Km}$ 522), porém resultaram infrutíferas, pois não foram obtidos espécimes nessas condiçōes. $\mathrm{Na}$ Tabela 1 , constam os resultados das coletas e os períodos em que os exemplares foram capturados durante todo o horário da mesma. Observcu-se uma variação no horário de início da atividade de picar nos diferentes pontos de coleta. A atividade iniciou-se mais cedo no Igarapé $\mathrm{Ca}$ nastra e no Ric Jauaperi (18:00 e 18:10 horas respectivamente). O término da atividade também variou, sendo mais tardia no Igarapé $\mathrm{Ca}$ nastra (21:00 horas). A duração atingiu, no máximo, duas horas, registradas também no referido Igarapé. O maior número de exemplares foi coletado no $\mathrm{Km} \mathrm{138}$, totalizando 245 espécimes em duas coletas. Verificou-se que a atividade de picar durante as 4 horas de observação está concentrada, na maoiria dos pontos de coleta, no horário das 18h:30' às 19h:10', aproximadamente.

Resultados semelhantes na atividade de picar de Anopheles nuneztovari também foram observados por Panday (1977) e Paraluppi (1978), respectivamente para populações de A. nuneztovari do Suriname e de Manaus. C primeiro autor, durante 24 horas de observac registrou um repentino pico de atividar no horário das 18:00 às 19:00 horas. Med' s detalhadas deste pico mostraram que ? se mais intensa está concentrada no horári 18:15-18:20 às $18 \mathrm{~h}: 45^{\prime}-18 \mathrm{~h}: 50^{\prime}$. Na popular so de Manaus, Paraluppi (1978) verificou o ' Jrário das 18h:30' às 19h:00. Bruyning (19r $/$ in Panday, 1977) também observou um píco de atividade logo após o anoitecer.

Estudos em insetos hematófagos têm mostrado que a tividade de picar é uma característica que pode ser influenciada por fatores intrínsecos e extrínsecos ao organismo; esta atividade pode ainda ser totalmente suprimida quando fatores físicos variam além de determinados limites. No entanto, apesar da existência de variações, um padrão característico pode ser verificado para cada espécie e uma mesma espécie pode wariar seu padrão ao longo de sua área de distribuição. Estudos em espécies de Sımulium (Diptera, Simuliidae) de duas lo- calidades brasileiras evidenciaram que $S$. simplicicolor do Arịuanã e $S$. guianense e $S$. sanguineum s.1. cio Aruá apresentam um padrão de atividade claramente bimodal; entretanto,

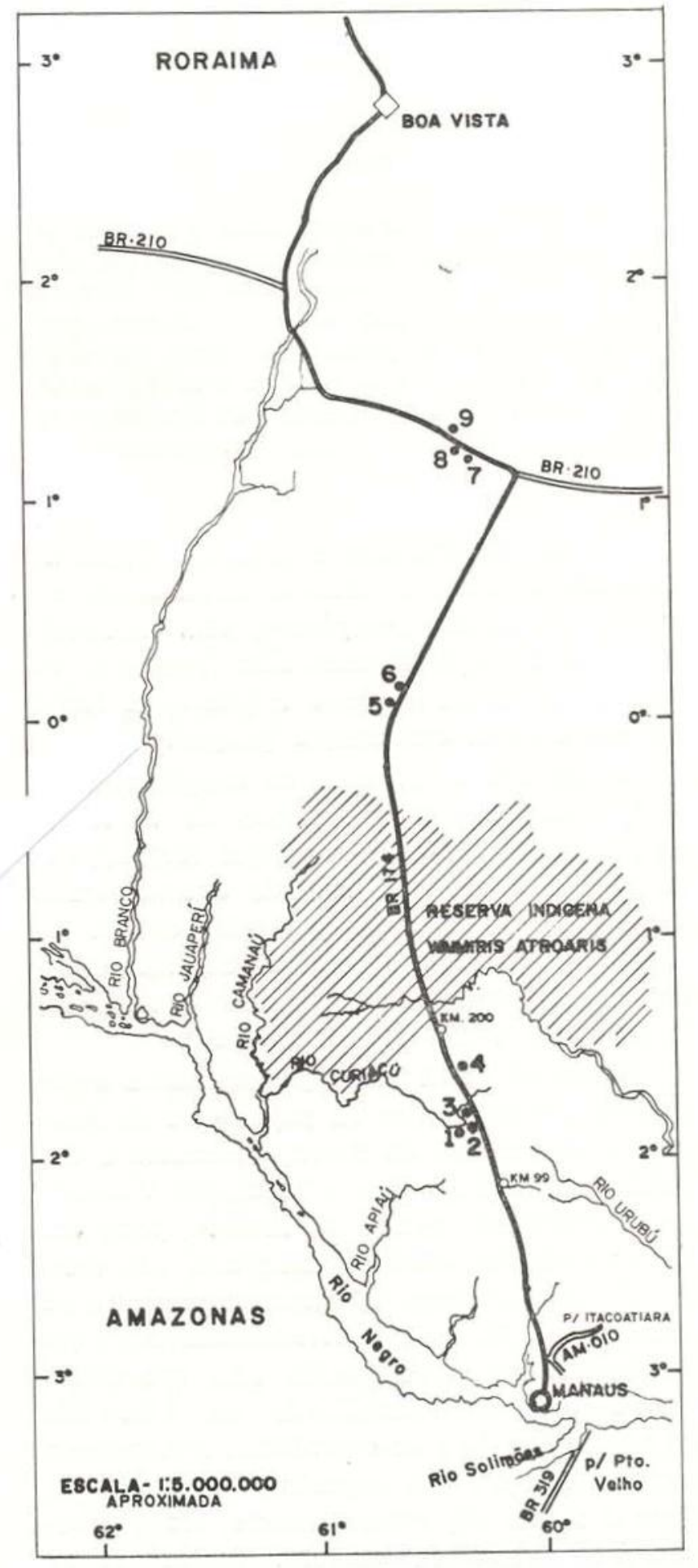

Fig. 1 - Localizaçăo dos pontos de coletas, ao longo da Rodovia BR-174. As denominações das localidades constam da Tabela 1. (Mapa segundo Ferraroni \& Hayes, 1979). 
S. sanguineum s.1. do Aripuanã mostrou um padrão com três picos de atividade (Lacey \& Charlwood, 1980). Diferenças no ciclo de picadas de Stomoxys calcitrans (Diptera, Muscidae) foram relacionadas à fêmeas com diferentes taxas de paridade (Charlwood \& Lopes, 1980). Em Anopheles darlingi foram verificadas variações geográficas quanto ao ciclo de picacias (Charlwood \& Hayes, 1978) e análise das taxas de paridade das fêmeas evidenciou que 0 número de adultos sobreviventes permanece baixo, apesar das modificações na densidade populacional (Charlwood, 1980). Anopheles nuneztovari, analisada neste trabalho, apresentou um padrão de atividade unimodal. com um pico no início da noite, que foi verif: do desde o $\mathrm{Km} 137$ ao $\mathrm{Km} 522$ (tabela 1) .e mesmo padrão, observado também pare populações de Manaus e do Suriname forme já mencionado, foi ainda notado re temente em populações de $A$. nuneztovari região de Tucuruí, Pará (Tadei, não publir o) .
O registro de populações exofílicas de Anopheles nuneztovari, assim como endofílicas, é de grande interesse do ponto de vista epidemiológico, considerando-se principalmente dois aspectos: um relacionado com o controle do vetor, 'em áreas onde $A$. nuneztovari é considerada transmissora da malária, e o outro relacionado com a possibilidade dessa espécie vir a ser um vetor secundário, em áreas em que normalmente não é vetora. Em relação ao primeiro, o caráter incontrolável da malária humana no oeste da Venezuela foi atribuído ao comportamento exofílico de A. nuneztovari (Gabaldon, 1969; Gabaldon et al., 1975). Quanto ao segundo, Panday (1977) mencionou que $A$. nuneztovari poderia estar envolvida na transmissão da malária no Suriname, considerando a escassez de $A$. darlingi e a ausência da mesma em capturas realizadas durante recentes surtos de malária, em certas áreas, onde A. nuneztovari foi registrada em altas densidades.

TABELA 1 - Freqüência, em núr os absolutos, e período de captura de Anopheles nuneztovari nos pontos de coleta ao longo da Rodo' BR-174, durante 4 horas de observação (18:00 às 22:00 horas)

\begin{tabular}{|c|c|c|c|}
\hline Localidades & $\begin{array}{l}\text { Período de } \\
\text { captura }\end{array}$ & $\begin{array}{l}\text { Duração da } \\
\text { atividade de picar }\end{array}$ & $\begin{array}{c}\text { Número de } \\
\text { exemplares coletados }\end{array}$ \\
\hline 1. Igarapé $\mathrm{Canas}^{+}$a. (Km 137) * & $\begin{array}{l}18: 00-19: 00 \\
20: 00-21: 00\end{array}$ & 2:00 & $\begin{array}{r}19 \\
3\end{array}$ \\
\hline 2. $\mathrm{Km} 138$ & $\begin{array}{l}18: 30-19: 40 \\
18: 35-19: 10 \\
19: 30-20: 40\end{array}$ & $\begin{array}{l}1: 10 \\
1: 45\end{array}$ & $\begin{array}{r}126 \\
106 \\
13\end{array}$ \\
\hline 3. $\mathrm{Km} 147$ & $18: 30-19: 05$ & $0: 35$ & 42 \\
\hline 4. $\mathrm{Km} 175$ & $18: 45-19: 10$ & $0: 25$ & 28 \\
\hline 5. Rio Jauaperi $(\mathrm{Km} \mathrm{420)}$ & $\begin{array}{l}18: 45-19: 30 \\
20: 00-20: 30\end{array}$ & $1: 15$ & $\begin{array}{r}19 \\
2\end{array}$ \\
\hline 6. Rio Jauaperi ( $\mathrm{Km} \mathrm{422)}$ & $18: 10-19: 00$ & $0: 50$ & 3 \\
\hline 7. Aeroporto da FAB (Km 513) & $18: 40-19: 10$ & $0: 30$ & 12 \\
\hline 8. $\mathrm{Km} 522$ & $\begin{array}{l}18: 45-19: 00 \\
19: 50-20: 45\end{array}$ & $1: 10$ & $\begin{array}{r}25 \\
2\end{array}$ \\
\hline 9. $\mathrm{Km} 530$ & $\begin{array}{l}18: 30-19: 10 \\
19: 30-20: 30\end{array}$ & $1: 40$ & $\begin{array}{l}4 \\
1\end{array}$ \\
\hline
\end{tabular}

\footnotetext{
(*) - Descendo o igarapé 5 ' km, mata adentro.
} 


\section{Agradecimentos}

Aos Professores Doutores Warwick Estevam Kerr e Hermione Elly Melara de Campos Bicudo pela leitura crítica do manuscrito.

\section{SUMMARY}

Observations on the biting activity of the Anopheles nuneztovari collected between $18: 00$ and 22:00 in different places along BR-174 Highway (Manaus/Boa Vista) which presented the exophilic habit are here related. Variations at the beginning and at the end of the biting activity were noted, but it is concentrated approximately between 18:30 and 19:10.

\section{REFERENCIAS BIBLIOGRÁFICAS}

CHARLWOOLD, J.D.

1980 - Observations on the bionomics of Anopheles darlingi Root (Diptera, Culicidae) from Brazil. Bull. Ent. Res., 70: 685-693.

CHARLWOOD, J.D. \& HAYES, J.

1978 - Variaçōes geográficas no ciclo de picada do Anopheles darlingi Root no Brasil. Acta Amazonica, 8 (4): 601-603.

CHARLWOOD, J.D. \& LOPES, J.

1980 - The age-structure and biting behaviour of Stomoxys calcitrans (L.) (Diptera: Muscidas) from Manaus, Brazil. Bull. Ent. Res., 70: $549-557$

DEANE, L.M.; CAUSEY, O.R.; DEANE, M.P.

1948 - Notas sobre a distribuição e a biologia dos Anofelinos das regiōes Nordestinas e Amazônica do Brasil. Rev. Serv. Esp. Saúde Pub., 1: 827-965.

ELLIOTT, R.

1968 - Studies on man-vector contact in sor nalarious areas in Colombia. Bull. WI flth. Org., 38: 239-253.

1972 - The influence of Vector Behavio' un Malaria Transmissor. Am. J. Trop. d. Hyg., 21: 755-763.

FERRARONI, J.J. \& HAYES, J.

1979 - Aspectos epidemiológicos ' malária no Amazonas. Acta Amazonice i: 471-479.
GABALDON, A.

1969 - Global malaria erradication: changes of strategy and future outlook. Am. J. Trop. Med. Hyg., 18: 641-656.

GABALDON, A. \& GUERRERO, L.

1959 - An attempt to eradicate by the weekly administration of purimethamine in areas of out-of-doors transmission in Venezuela. Am. J. Trop. Med. Hyg., 8: 433-439.

GABALDON, A.; GUERRERO, L.; MARTIN, G.G.

1963 - Malaria refractaria en el occidente de Venezuela. Rev. Venez. Sanid., 14: 513-530.

GABALDON, A.; MARTIN, G.G.; SIFONTES, R.

1975 - Necesidades em el campo de la investigación del Programa Nacional de Erradicación y Control de la Malaria de Venezuela. Bol. Dir. Malariol. y San. Amb., 15 (6): 263-285.

GARCIA-MARTIN, G.

1955 - Problemas entomológicos que dificultan la erradicación de la malaria en el Estado Zulia. Trabajo presentado em el XI Congresso Venezolano de Ciencias Medicas. Caracas.

KITZMILLER, J.B.; KREUTZER, R.D.; TALLAFERRO, E.

1973 - Chromosomal differences in populations of Anopheles nuneztovari. Bull. Wld. Hlth. Org. 48: $435-455$.

LACEY, L.A. \& CHARLWOOD, J.D.

1980 - On the biting activities of some anthropophilic Amazonian Simuliidae (Diptera). Bull. Ent. Res., 70: 495-509.

PANDAY, R.S.

1977 - Anopheles nuneztovari and malaria transmission in Surinam. Mosquito News, 37: 728-737.

PARALUPPI, N.D.

1978 - Alguns aspectos da Biologia e do comportamento do Anopheles $(\mathrm{N})$ nuneztovari Gabaldon (Diptera, Culicidae), dos arredores da cidade de Manaus, Amazonas, Brasil. Dissertação de Mestrado. Instituto Nacional de Pesquisas da Amazônia e Fundação Universidade do Amazonas.

STEINER, W.W.M.; KITZMILLER, J.B.; OSTERBUR, D.L. 1980 - Gene differentiation in chromosome races of Anopheles nuneztovari (Gabaldon). Mosquito Systematics, 12: 306-319.

(Aceito para publicação em 28/08/81) 